\title{
Applications of Unitary Symmetry and Combinatorics
}


This page intentionally left blank 


\section{Applications of \\ Unitary Symmetry and Combinatorics}

\section{James D Louck}

Los Alamos National Laboratory Fellow

Santa Fe, New Mexico, USA 


\section{Published by}

World Scientific Publishing Co. Pte. Ltd.

5 Toh Tuck Link, Singapore 596224

USA office: 27 Warren Street, Suite 401-402, Hackensack, NJ 07601

UK office: 57 Shelton Street, Covent Garden, London WC2H 9HE

\section{British Library Cataloguing-in-Publication Data}

A catalogue record for this book is available from the British Library.

\section{APPLICATIONS OF UNITARY SYMMETRY AND COMBINATORICS}

Copyright $(92011$ by World Scientific Publishing Co. Pte. Ltd.

All rights reserved. This book, or parts thereof, may not be reproduced in any form or by any means, electronic or mechanical, including photocopying, recording or any information storage and retrieval system now known or to be invented, without written permission from the Publisher.

For photocopying of material in this volume, please pay a copying fee through the Copyright Clearance Center, Inc., 222 Rosewood Drive, Danvers, MA 01923, USA. In this case permission to photocopy is not required from the publisher.

ISBN-13 978-981-4350-71-6

ISBN-10 981-4350-71-0

Printed in Singapore. 
In recognition of contributions to the generation and spread of knowledge

\author{
William Y. C. Chen \\ Tadeusz and Barbara Lulek \\ And to the memory of \\ Lawrence C. Biedenharn \\ Gian-Carlo Rota
}


This page intentionally left blank 


\section{Preface and Prelude}

We have titled this monograph "Applications of Unitary Symmetry and Combinatorics" because it uses methods developed in the earlier volume "Unitary Symmetry and Combinatorics," World Scientific, 2008 (hereafter Ref. [46] is referred to as [L]). These applications are highly topical, and come in three classes: (i) Those still fully mathematical in content that synthesize the common structure of doubly stochastic, magic square, and alternating sign matrices by their common expansions as linear combinations of permutation matrices; (ii) those with an associated physical significance such as the role of doubly stochastic matrices and complete sets of commuting Hermitian operators in the probabilistic interpretation of nonrelativistic quantum mechanics, the role of magic squares in a generalization of the Regge magic square realization of the domains of definition of the quantum numbers of angular momenta and their counting formulas (Chapter 6), and the relation between alternating sign matrices and a class of Gelfand-Tsetlin patterns familiar from the representation of irreducible representations of the unitary groups (Chapter 7) and their counting formulas; and (iii) a physical application to the diagonalization of the Heisenberg magnetic ring Hamiltonian, viewed as a composite system in which the total angular momentum is conserved.

A uniform viewpoint of rotations is adopted at the outset from [L], based on the method of Cartan [15] (see also [6]), where it is fully defined and discussed. It is not often made explicit in the present volume:

$A$ unitary rotation of a composite system is its redescription under an $S U(2)$ unitary group frame rotation of a right-handed triad of perpendicular unit vectors $\left(\mathbf{e}_{1}, \mathbf{e}_{2}, \mathbf{e}_{3}\right)$ that serves as common reference system for the description of all the constituent parts of the system.

This Preface serves three purposes: A prelude and synthesis of things to come based on results obtained in [L], now focused strongly on the basic structural elements and their role in bringing unity to the understanding of the angular momentum properties of complex systems viewed as composite wholes; a summary of the contents by topics; and the usual elements of style, readership, acknowledgments, etc. 


\section{OVERVIEW AND SYNTHESIS OF BINARY COUPLING THEORY}

The theory of the binary coupling of $n$ angular momenta is about the pairwise addition of $n$ angular momenta associated with $n$ constituent parts of a composite physical system and the construction of the associated state vectors of the composite system from the $S U(2)$ irreducible angular momentum multiplets of the parts. Each such possible way of effecting the addition is called a binary coupling scheme.

We set forth in the following paragraphs the underlying conceptual basis of such binary coupling scheme. Each binary coupling scheme of order $n$ may be described in terms of a sequence having two types of parts: $n$ points $\circ \circ \cdots \circ$ and $n-1$ parenthesis pairs ()$,(), \ldots,($ ). A parenthesis pair ( ) constitutes a single part. Thus, the number of parts in the full sequence is $2 n-1$. By definition, the binary bracketing of order 1 is $\circ$ itself, the binary bracketing of order 2 is (o०), the two binary bracketings of order 3 are $((\circ \circ))$ and $(\circ(\circ \circ)), \ldots$ In general, we have the definition:

$A$ binary bracketing $\mathbb{B}_{n}$ of order $n \geq 2$ is any sequence in the $n$ points $\circ$ and the $n-1$ parenthesis pairs ( ) that satisfies the two conditions: (i) It contains a binary bracketing of order 2 , and (ii) the mapping (००) $\mapsto \circ$ gives a binary bracketing of order $n-1$.

Then, since the mapping $(\circ \circ) \mapsto \circ$ again gives a binary bracketing for $n \geq 3$, the new binary bracketing of order $n-1$ again contains a binary bracketing of order 2 . This implies that this mapping property can be used repeatedly to reduce every binary bracketing of arbitrary order to $\circ$, the binary bracketing of order 1 .

The appropriate mathematical concept for diagramming all such binary bracketings of order $n$ is that of a binary tree of order $n$. We have described in [L] a "bifurcation of points" build-up principle for constructing the set $\mathbb{T}_{n}$ of all binary trees of order $n$ in terms of levels (see [L, Sect. 2.2]). This is a standard procedure found in many books on combinatorics. It can also be described in terms of an assembly of four basic objects called forks that come in four types, as enumerated by

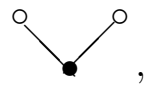

(1)

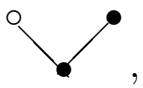

(2)

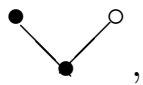

(3)

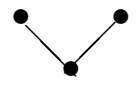

(4)

The $\bullet$ point at the bottom of these diagrams is called the root of the fork, and the other two point are called the endpoints of the fork. The assembly rule for forks into a binary tree of order $n$ can be formulated in term of the "pasting" together of forks. 
Our interest in viewing a binary tree as being composed of a collection of pasted forks of four basic types is because the configuration of forks that appears in the binary tree encode exactly how the pairwise addition of angular momenta of the constituents of a composite system is to be effected. Each such labeled fork has associated with it the elementary rule of addition of the two angular momenta, as well as the WignerClebsch-Gordan (WCG) coefficients $C_{m_{1}}^{j_{1}} j_{2}{ }_{2} k$ that effect the coupling of the state vectors of two subsystems of a composite system having angular momentum $\mathbf{J}(1)$ and $\mathbf{J}(2)$, respectively, to an intermediate angular momentum $\mathbf{J}(1)+\mathbf{J}(2)=\mathbf{K}$, as depicted by the labeled fork:

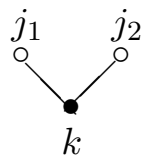

$$
k=j_{1}+j_{2}, j_{1}+j_{2}-1, \ldots,\left|j_{1}-j_{2}\right|
$$

Similarly, the labeled basic fork 2 given by

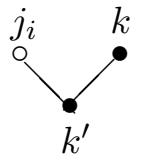

$$
k^{\prime}=j_{i}+k, j_{i}+k-1, \ldots,\left|j_{i}-k\right|
$$

encodes the addition $\mathbf{J}(i)+\mathbf{K}=\mathbf{K}^{\prime}$ of an angular momentum $\mathbf{J}(i)$ of the constituent system and an intermediate angular momentum $\mathbf{K}$ to a "total" intermediate angular momentum $\mathbf{K}^{\prime}$, as well as the attended

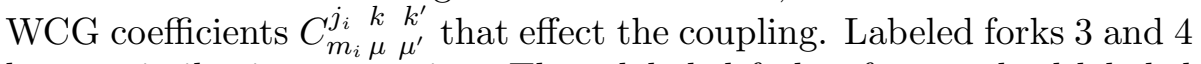
have a similar interpretation. These labeled forks of a standard labeled binary tree of order $n$ encode the constituent angular momenta that enter into the description of the basic $S U(2)$ irreducible state vectors of a composite system.

A build-up rule for the pasting of forks can be described as follows:

1. Select a fork from the set of four forks above and place the $\bullet$ root point over any $\bullet$ endpoint of the four forks, merging the two $\bullet$ points to a single $\bullet$ point. Repeat this pasting process for each basic fork. This step gives a set of seventeen distinct graphs, where we include the basic fork containing the two $\circ$ points in the collection: 

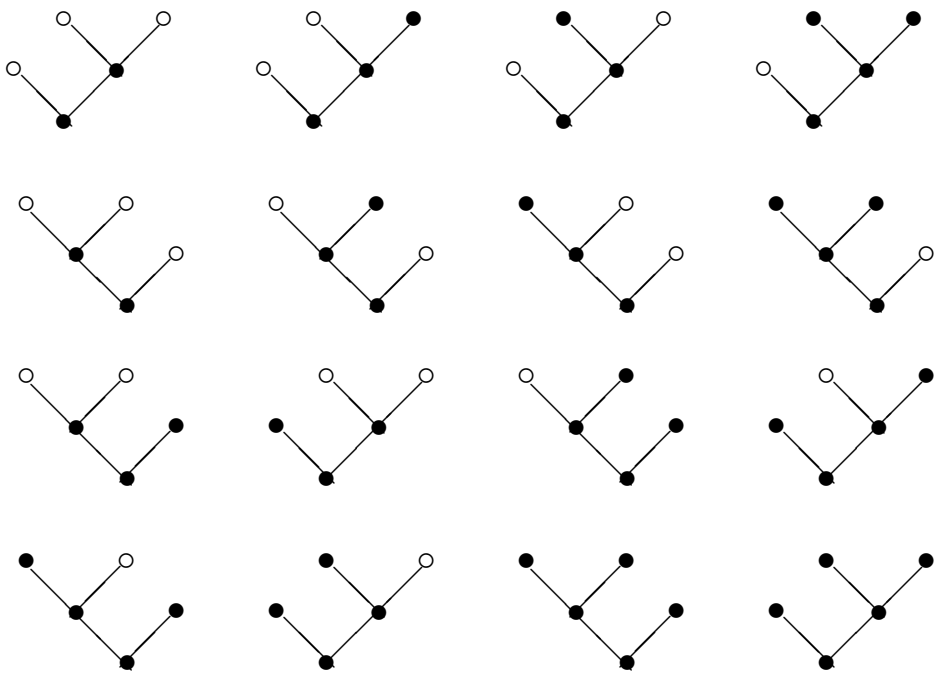

2. Select a single graph from the set of seventeen graphs generated at Step 1 and repeat the pasting process with each of the four basic forks. This gives back the three graphs from the collection above having only endpoints of type $\circ$, which includes all binary tree graphs of order 2 and order 3 , and, in addition, ninety-six more graphs as follows: (1)(4)(6) from the six graphs above having one • endpoints; (2)(4)(6) from the six graphs having two • endpoints, and (3)(4)(2) from the three graphs having three $\bullet$ endpoints. From this large collection, put aside those having only $\circ$ endpoints, including no repetitions. This gives the set of all binary trees or order $2,3,4$ :
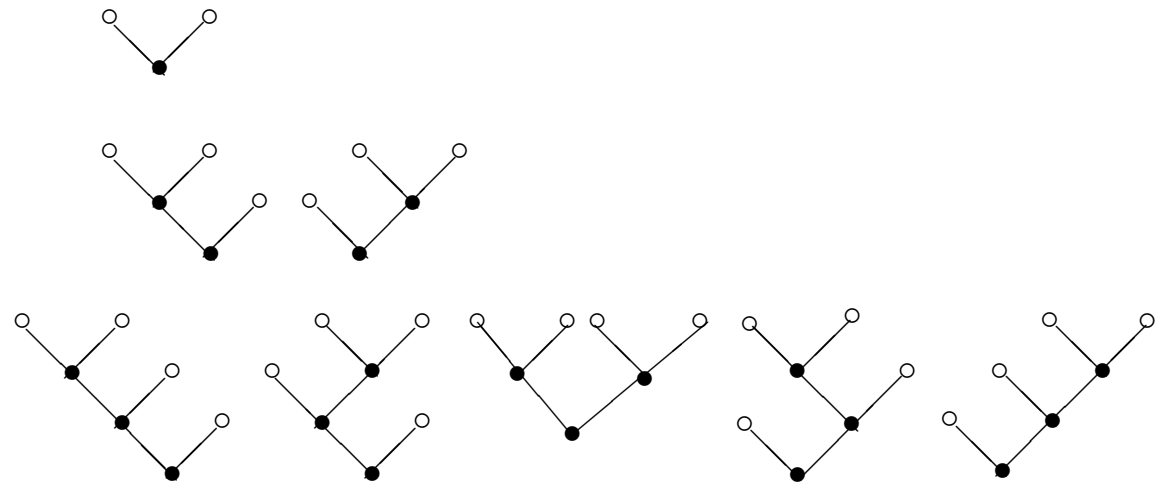
3. Select a single graph from the full set generated at Step 2 and repeat the pasting process. putting aside all those having only $\circ$ endpoints, including no repetitions. Repeat this process for the next set of graphs, etc. At Step $h$ of this pasting process of basic forks to the full collection generated at Step $h-1$, there is obtained a huge multiset of graphs that includes all binary trees of order $2,3, \ldots, h+2$ - all those with $\circ$ endpoints. A very large number of graphs with $1 \bullet$ endpoint, $2 \bullet$ endpoints, ... is also obtained, these being needed for the next step of the pasting process. All binary trees of order $n$ are included in the set of graphs generated by the pasting process at step $h=n-2$, these being the ones put aside.

The pasting process is a very inefficient method for obtaining the set of binary trees of order $n$. A more efficient method is to generate this subset recursively by the following procedure: Suppose the set of binary trees of order $n$ has already been obtained, and that it contains $a_{n}$ members. Then, consider the new set of $n a_{n}$ graphs obtained by replacing a single $\circ$ endpoint by a $\bullet$ point in each of the $a_{n}$ binary tree graphs. In the next step, paste the $\bullet$ root of basic fork 1 over each of these $\bullet$ endpoints, thus obtaining $n a_{n}$ binary trees of order $n+1$. This multiset of cubic graphs of order $n+1$ then contains the set of $a_{n+1}$ binary trees of order $n+1$. Thus, starting with fork 1 from the basic set of four, we generate all binary trees of arbitrary order by this pasting procedure. (This procedure works because every graph having one • endpoint is included in the huge set generate in Items $1,2,3$ above.)

Only binary trees having o endpoints enter into the binary coupling theory of angular momenta because each such coupling scheme must correspond to a binary bracketing. The set of binary bracketings of order $n$ is one-to-one with the set of binary trees of order $n$, and the bijection rule between the two sets can be formulated exactly. The cardinality of the set $\mathbb{T}_{n}$ is the Catalan number $a_{n}$. We repeat, for convenience, many examples of such binary bracketings and corresponding binary trees from $[\mathrm{L}]$, and also give in this volume many more, including examples for arbitrary $n$. As in $[\mathrm{L}]$, we call the binary bracketing corresponding to a given binary tree the shape of the binary tree:

The shape $S h_{T}$ of a binary tree $T \in \mathbb{T}_{n}$ is defined to be the binary bracketing of order $n$ corresponding to $T$.

The concept of shape transformation is so basic that we discuss it here in the Preface in its simplest realization: There are two binary trees of order three, as given above. We now label the endpoints of these binary trees by a permutation of some arbitrary set of elements $x_{1}, x_{2}, x_{3}$ : 


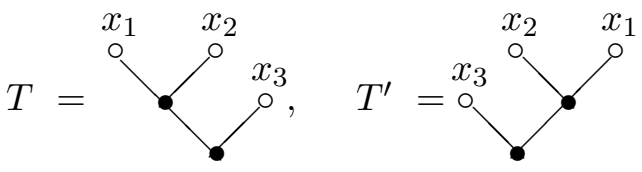

We initially ignore the labels. The first graph corresponds to the binary bracketing $((\circ \circ) \circ)$, and the second one to $(\circ(\circ \circ))$. The binary bracketing, now called the shape of the binary tree, is defined in the obvious way by reading left-to-right across the binary tree, and inserting a parenthesis pair for each • point:

$$
\mathrm{Sh}_{T}=((\circ \circ) \circ), \quad \mathrm{Sh}_{T^{\prime}}=(\circ(\circ \circ)) .
$$

These shapes are called unlabeled shapes. If the $\circ$ endpoints are labeled by a permutation of distinct symbols such as $x_{1}, x_{2}, x_{3}$, the $\circ$ points of the shape are labeled by the corresponding symbols to obtain the labeled shapes

$$
\operatorname{Sh}_{T}\left(x_{1}, x_{2}, x_{3}\right)=\left(\left(x_{1} x_{2}\right) x_{3}\right), \quad \operatorname{Sh}_{T^{\prime}}\left(x_{2}, x_{1}, x_{3}\right)=\left(x_{2}\left(x_{1} x_{3}\right)\right) .
$$

What is interesting now is that the first labeled shape can be transformed to the second labeled shape by the elementary operations $C$ and $A$ of commutation and association:

$$
\left(\left(x_{1} x_{2}\right) x_{3}\right) \stackrel{C}{\longrightarrow}\left(\left(x_{2} x_{1}\right) x_{3}\right) \stackrel{A}{\longrightarrow}\left(x_{2}\left(x_{1} x_{3}\right)\right),
$$

where the action of commutation $C$ and that of association $A$ have their usual meaning:

$$
C:(x y) \mapsto(y x), \quad A:(x y) z \mapsto x(y z) .
$$

Thus, we have the shape transformation:

$$
\operatorname{Sh}_{T}\left(x_{1}, x_{2}, x_{3}\right) \stackrel{A C}{\longrightarrow} \operatorname{Sh}_{T^{\prime}}\left(x_{2}, x_{1}, x_{3}\right),
$$

where the convention for the action of commutation and association is that $C$ acts first on the shape $\operatorname{Sh}_{T}\left(x_{1}, x_{2}, x_{3}\right)$ to effect the transformation to the shape $\operatorname{Sh}_{T}\left(x_{2}, x_{1}, x_{3}\right)$ followed by the action of $A$ on $\operatorname{Sh}_{T}\left(x_{2}, x_{1}, x_{3}\right)$ to give the shape $\mathrm{Sh}_{T^{\prime}}\left(x_{2}, x_{1}, x_{3}\right)$.

We state at the outset the generalization of this result:

The set $\mathbb{T}_{n}$ of binary trees of order $n$ is unambiguously enumerated by its set of shapes $S h_{T}, T \in \mathbb{T}_{n}$, the number of which is given by the Catalan numbers $a_{n}$. Let $x_{1}, x_{2}, \ldots, x_{n}$ be a collection of $n$ arbitrary distinct objects and $x_{\pi}=\left(x_{\pi_{1}}, x_{\pi_{2}}, \ldots, x_{\pi_{n}}\right)$ an arbitrary permutation of the $x_{i}$, where $\pi=\left(\pi_{1}, \pi_{2}, \ldots, \pi_{n}\right)$ is an arbitrary permutation in the group $S_{n}$ of all permutations of the reference set $1,2, \ldots, n$. 
Then, there exists a shape transformation $w(A, C)$ such that the labeled shape $S h_{T}\left(x_{\pi}\right)$ is transformed to the labeled shape $S h_{T^{\prime}}\left(x_{\pi^{\prime}}\right)$, for each $T \in \mathbb{T}_{n}, \pi \in S_{n}, T^{\prime} \in \mathbb{T}_{n}, \pi^{\prime} \in S_{n}$; that is,

$$
S h_{T}\left(x_{\pi}\right) \stackrel{w(A, C)}{\longrightarrow} S h_{T^{\prime}}\left(x_{\pi^{\prime}}\right),
$$

where the shape transformation $w(A, C)$ is a word in the two letters $A$ and $C$.

The content of this result will be amplified by many explicit examples. It is a basic unifying result for the binary theory of the coupling of $n$ angular momenta.

The pairwise addition of $n$ angular momenta $\mathbf{J}(i)=J_{1}(i) \mathbf{e}_{1}+J_{2}(i) \mathbf{e}_{2}+$ $J_{3}(i) \mathbf{e}_{3}, i=1,2, \ldots, n$, with components referred to a common righthanded inertial reference frame $\left(\mathbf{e}_{1}, \mathbf{e}_{2}, \mathbf{e}_{3}\right)$, to a total angular momentum $\mathbf{J}=J_{1} \mathbf{e}_{1}+J_{2} \mathbf{e}_{2}+J_{3} \mathbf{e}_{3}=\mathbf{J}(1)+\mathbf{J}(2)+\cdots+\mathbf{J}(n)$ is realized in all possible ways by the standard labeling of each binary tree $T \in \mathbb{T}_{n}$ as given in terms of its shape by $S h_{T}\left(\mathbf{J}\left(\pi_{1}\right), \mathbf{J}\left(\pi_{2}\right), \ldots, \mathbf{J}\left(\pi_{n}\right)\right)$, where $\pi=\left(\pi_{1}, \pi_{2}, \ldots, \pi_{n}\right)$ is any permutation $\pi \in S_{n}$. The pairwise addition encoded in a given binary tree for any given permutation $\pi \in S_{n}$ is called a coupling scheme. For example, the two coupling schemes encoded by the shapes of the binary trees of order 3 given above are:

$$
((\mathbf{J}(1)+\mathbf{J}(2))+\mathbf{J}(3)), \quad(\mathbf{J}(3)+(\mathbf{J}(2)+\mathbf{J}(1))) .
$$

For general $n$, there are $n ! a_{n}$ distinct coupling schemes for the pairwise addition of $n$ angular momenta. The rule whereby we assign the $n$ angular momenta $(\mathbf{J}(1), \mathbf{J}(2), \ldots, \mathbf{J}(n))$ of the $n$ constituents of a composite system to the endpoints of a binary tree $T \in \mathbb{T}_{n}$ by the left-to-right assignment to the corresponding points in the shape $S h_{T}$ of $T$ is called the standard rule labeling of the o points (all endpoints) of the binary tree $T$. In many instances, we need also to apply this standard rule labeling for the assignment of a permutation $\left(\mathbf{J}\left(\pi_{1}\right), \mathbf{J}\left(\pi_{2}\right), \ldots, \mathbf{J}\left(\pi_{n}\right)\right)$ of the angular momenta of the constituent parts, as illustrated in the example above. Caution must be exercised in the interpretation of all these pairwise additions of angular momenta in terms of the tensor product space in which these various angular momenta act. This is reviewed from $[\mathrm{L}]$ in Chapters 1 and 5 in the context of the applications made here. A standard labeling rule is required to define unambiguously objects such as Wigner-Clebsch-Gordan coefficients and triangle coefficients to labeled binary trees, objects that given numerical content to the theory.

To summarize: In the context of the binary coupling theory of angular momenta, we deal with standard labeled binary trees, their shapes, and the transformations between shapes. Out these few simple underlying structural elements there emerges a theory of almost unlimited, 
but manageable, complexity: Mathematically, it is theory of relations between $3(n-1)-j$ coefficients and Racah coefficients and their cubic graphs; physically, it is a theory of all possible ways to compound, pairwise, the individual angular momenta of the $n$ constituent parts of a complex composite system to the total angular momentum of the system.

The implementation of the binary coupling theory of angular momenta leads directly to the Dirac concept [24] of characterizing the Hilbert space state vectors of each coupling scheme in terms of complete sets of commuting Hermitian operators. This characterization is described in detail in [L], and reviewed in the present volume in Chapters 1 and 5; it is described broadly as follows. The complete set of $2 n \mathrm{mu}-$ tually commuting Hermitian operators for each coupling scheme $T \in \mathbb{T}_{n}$ is given by

$$
\begin{aligned}
& \mathbf{J}^{2}(1), \mathbf{J}^{2}(2), \ldots, \mathbf{J}^{2}(n), \mathbf{J}^{2}, J_{3} ; \\
& \mathbf{K}_{T}^{2}(1), \mathbf{K}_{T}^{2}(2), \ldots, \mathbf{K}_{T}^{2}(n-2) .
\end{aligned}
$$

The first line of operators consists of the $n$ total angular momentum squared of each of the constituent systems, together with the total angular momentum squared of the composite system and its 3-component. The second line of operators consists of the squares of the $n-2$ so-called intermediate angular momenta, $\mathbf{K}_{T}(i)=\left(K_{T, 1}(i), K_{T, 2}(i), K_{T, 3}(i)\right), i=$ $1,2, \ldots, n-2$. There is a distinct set of such intermediate angular momenta associated with each binary tree $T \in \mathbb{T}_{n}$, where each $\mathbf{K}_{T}(i)$ is a $0-1$ linear combination of the constituent angular momenta $\mathbf{J}(i), i=$ $1,2, \ldots, n$, in which the 0 and 1 coefficients are uniquely determined by the shape of the binary tree $T \in \mathbb{T}_{n}$. Thus, each standard labeled binary coupling scheme has associated with it a unique complete set of $2 n$ mutually commuting Hermitian operators, as given generally above. For example, the complete sets of mutually commuting Hermitian operators associated with the labeled binary trees $T$ and $T^{\prime}$ of order 3 given above are the following, respectively:

$$
\begin{aligned}
& \text { scheme } T: \mathbf{J}^{2}(1), \mathbf{J}^{2}(2), \mathbf{J}^{2}(3), \mathbf{J}^{2}, J(3) ; \mathbf{K}_{T}^{2}(1)=(\mathbf{J}(1)+\mathbf{J}(2))^{2} . \\
& \text { scheme } T^{\prime}: \mathbf{J}^{2}(1), \mathbf{J}^{2}(2), \mathbf{J}^{2}(3), \mathbf{J}^{2}, J(3) ; \mathbf{K}_{T^{\prime}}^{2}(1)=(\mathbf{J}(2)+\mathbf{J}(1))^{2} .
\end{aligned}
$$

There is a set of simultaneous eigenvectors associated with each complete set of mutually commuting Hermitian operators defined above for each binary tree $T \in \mathbb{T}_{n}$ of labeled shape $\operatorname{Sh}_{T}\left(\mathbf{J}\left(\pi_{1}\right), \mathbf{J}\left(\pi_{2}\right), \ldots, \mathbf{J}\left(\pi_{n}\right)\right)$. It is convenient now to denote this shape by $S h_{T}\left(\mathbf{j}_{\pi}\right)$, where we use the angular momentum quantum number $j_{i}$ in place of the angular momentum operator $\mathbf{J}(i)$ in the labeled shape. These are the quantum numbers $j_{i}$ associated with the eigenvalue $j_{i}\left(j_{i}+1\right)$ of the squared "total" angular momentum $\mathbf{J}^{2}(i)$, each $i=1,2, \ldots, n$, of each of the $n$ constituents of the 
composite physical system in question. The simultaneous eigenvectors in this set are denoted in the Dirac ket-vector notation by

$$
\left|T\left(\mathbf{j}_{\pi} \mathbf{k}\right)_{j m}\right\rangle
$$

where $\mathbf{j}_{\pi}=\left(j_{\pi_{1}}, j_{\pi_{2}}, \ldots, j_{\pi_{n}}\right)$, and where $j$ denotes the total angular momentum quantum number of the eigenvalue $j(j+1)$ of the squared total angular momentum $\mathbf{J}^{2}$ and $m$ the eigenvalue of the 3 -component $J_{3}$. We generally are interested in the finite set of vectors enumerated, for specified $\mathbf{j}=\left(j_{1}, j_{2}, \ldots, j_{n}\right)$, each $j_{i} \in\{0,1 / 2,1,3 / 2,2, \ldots\}$, by the range of values of the total angular momentum quantum numbers $j m$; and by the range of values of all the intermediate quantum numbers $\mathbf{k}=\left(k_{1}, k_{2}, \ldots, k_{n-2}\right)$. The latter are associated with the eigenvalues $k_{i}\left(k_{i}+1\right)$ of the squared intermediate angular momenta for given $T \in$ $\mathbb{T}_{n}$. This gives the set of eigenvectors associated with the labeled shape $S h_{T}\left(\mathbf{j}_{\pi}\right)$, and defines an orthonormal basis of a finite-dimensional tensor product Hilbert space denoted by $\mathcal{H}_{\mathbf{j}}=\mathcal{H}_{\mathbf{j}_{1}} \otimes \mathcal{H}_{\mathbf{j}_{2}} \otimes \cdots \otimes \mathcal{H}_{\mathbf{j}_{n}}$ of dimension equal to $\left(2 j_{1}+1\right)\left(2 j_{2}+1\right) \cdots\left(2 j_{n}+1\right)$. The domain of definition of $j m$ for each coupled state vector $\left|T\left(\mathbf{j}_{\pi} \mathbf{k}\right)_{j m}\right\rangle$ corresponding to the binary tree $T$ of shape $S h_{T}\left(\mathbf{j}_{\pi}\right)$ is $j \in\left\{j_{\min }, j_{\min }+1, \ldots, j_{\max }\right\}$, where $j_{\min }$ is the least nonnegative integer or positive half-odd integer among the $2^{n}$ sums of the form $\pm j_{1} \pm j_{2} \pm \cdots \pm j_{n}$, and $j_{\max }=j_{1}+j_{2}+\cdots+j_{n}$. The domain of definition of the intermediate quantum number $k_{i}$ depends on the labeled shape of the binary tree $T$; it belongs to a uniquely defined set as given by $\mathbf{k} \in \mathbb{K}_{T}^{(j)}\left(\mathbf{j}_{\pi}\right)$, the details of which are not important here. Thus, an orthonormal basis of the space $\mathcal{H}_{\mathbf{j}}$ is given, for each $T \in \mathbb{T}_{n}$ and each labeled shape $S h_{T}\left(\mathbf{j}_{\pi}\right)$, by

$$
\begin{gathered}
\mathbf{B}_{T}\left(\mathbf{j}_{\pi}\right)=\left\{\left|T\left(\mathbf{j}_{\pi} \mathbf{k}\right)_{j m}\right\rangle \mid j \in\left\{j_{\min }, j_{\min }+1, \ldots, j_{\max }\right\} ; \text { and for each } j,\right. \\
\mathbf{k} \in \mathbb{K}_{T}^{(j)}\left(\mathbf{j}_{\pi}\right) ; m \in\{j, j-1, \ldots,-j\} .
\end{gathered}
$$

In all, we have $n ! a_{n}$ sets of coupled orthonormal basis vectors, each set $\mathbf{B}_{T}\left(\mathbf{j}_{\pi}\right)$ giving a basis of the same tensor product space $\mathcal{H}_{\mathbf{j}}$.

We digress a moment to recall that the basic origin of the tensor product Hilbert space $\mathcal{H}_{\mathbf{j}}$ is just the vector space formed from the tensor product of the individual Hilbert spaces $\mathcal{H}_{\mathbf{j}_{i}}$ of dimension $2 j_{i}+1$ on which the angular momentum $\mathbf{J}(i)$ has the standard action, with the commuting Hermitian operators $\mathbf{J}^{2}(i), J_{3}(i)$ being diagonal with eigenvalues $j_{i}\left(j_{i}+1\right)$ and $m_{i}$. The orthonormal basis of the space $\mathcal{H}_{\mathbf{j}}$ is now the uncoupled basis $|\mathbf{j} \mathbf{m}\rangle=\left|j_{1} m_{1}\right\rangle \otimes\left|j_{2} m_{2}\right\rangle \otimes \cdots \otimes\left|j_{n} m_{n}\right\rangle$, in which we can have $j_{i} \in$ $\{0,1 / 2,1,3 / 2,2, \ldots\}$, and, for each selected $j_{i}$, the so-called projection quantum number $m_{i}$ assumes all values $m_{i}=j_{i}, j_{i}-1, \ldots,-j_{i}$. Thus, the uncoupled orthonormal basis $\mathbf{B}_{\mathbf{j}}=\left\{|\mathbf{j} \mathbf{m}\rangle \mid\right.$ each $\left.m_{i}=j_{i}, j_{i}-1, \ldots,-j_{i}\right\}$ of $\mathcal{H}_{\mathbf{j}}$ is the space of simultaneous eigenvectors of the complete mutually commuting Hermitian operators $\mathbf{J}^{2}(i), J_{3}(i), i=1,2, \ldots, n$, where 
the components of each of the angular momentum operators $\mathbf{J}(i)$ has the standard action on $\mathcal{H}_{\mathbf{j}_{i}}$. These basic relations are presented in great detail in Ref. [6], in [L], and reviewed in Chapter 1 of this volume. The important point for the present work is:

Each simultaneous eigenvector $\left|T\left(\mathbf{j}_{\pi} \mathbf{k}\right)_{j m}\right\rangle$ of the set of $2 n$ mutually commuting Hermitian operators corresponding to each binary tree $T \in \mathbb{T}_{n}$ of shape $\mathrm{Sh}_{T}\left(\mathbf{j}_{\pi}\right)$ is a real orthogonal transformation of the eigenvectors $|\mathbf{j} \mathbf{m}\rangle \in \mathbf{B}_{\mathbf{j}}$. The coefficients in each such transformation are generalized Wigner-Clebsch-Gordan (WCG) coefficients, which themselves are a product of known ordinary WCG coefficients, where the product is uniquely determined by the shape of the labeled binary tree $S h_{T}\left(\mathbf{j}_{\pi}\right)$.

This is, of course, just the expression of the property that we have constructed $n ! a_{n}$ uniquely defined coupled orthonormal basis sets of the space $\mathcal{H}_{\mathbf{j}}$ from the uncoupled basis of the same space $\mathcal{H}_{\mathbf{j}}$.

There is a class of subspaces of $\mathcal{H}_{\mathbf{j}}$ of particular interest for the present work. This class of subspaces is obtained from the basis vectors $\mathbf{B}_{T}\left(\mathbf{j}_{\pi}\right)$ of $\mathcal{H}_{\mathbf{j}}$ given above by selecting from the orthonormal basis vectors $\left|T\left(\mathbf{j}_{\pi} \mathbf{k}\right)_{j m}\right\rangle$ those that have a prescribed total angular momentum quantum number $j \in\left\{j_{\min }, j_{\min }+1, \ldots, j_{\max }\right\}$ and, for each such $j$, a prescribed projection quantum number $m \in\{j, j-1, \ldots,-j\}$. Thus, the orthonormal basis vectors in this set are given by

$$
\mathbf{B}_{T}\left(\mathbf{j}_{\pi}, j, m\right)=\left\{\left|T\left(\mathbf{j}_{\pi} \mathbf{k}\right)_{j m}\right\rangle \mid \text { each } \mathbf{k} \in \mathbb{K}_{T}^{(j)}\left(\mathbf{j}_{\pi}\right)\right\} .
$$

We further specialize this basis set to the case $\pi=$ identity permutation:

$$
\mathbf{B}_{T}(\mathbf{j}, j, m)=\left\{\left|T(\mathbf{j} \mathbf{k})_{j m}\right\rangle \mid \text { each } \mathbf{k} \in \mathbb{K}_{T}^{(j)}(\mathbf{j})\right\} .
$$

This basis set of orthonormal vectors then defines a subspace $\mathcal{H}(\mathbf{j}, j, m) \subset$ $\mathcal{H}_{\mathbf{j}}$, which is the same vector space for each $T \in \mathbb{T}_{n}$ and for every permutation $\mathbf{j}_{\pi}$ of $\mathbf{j}$; that is, the following direct sum decomposition of the tensor product space $\mathcal{H}_{j}$ holds:

$$
\mathcal{H}_{\mathbf{j}}=\sum_{j=j_{\min }}^{j_{\max }} \sum_{m=-j}^{j} \oplus \mathcal{H}(\mathbf{j}, j, m) .
$$

We repeat: The important structural result for this vector space decomposition is:

Each basis set $\mathbf{B}_{T}\left(\mathbf{j}_{\pi}, j, m\right), T \in \mathbb{T}_{n}, \pi \in S_{n}$, is an orthonormal basis of one and the same space $\mathcal{H}(\mathbf{j}, j, m)$.

The dimension of the tensor product space $\mathcal{H}(\mathbf{j}, j, m) \subset \mathcal{H}_{\mathbf{j}}$ is $N_{j}(\mathbf{j})$, the Clebsch-Gordan (CG) number. This important number is the number of times that a given $j \in\left\{j_{\min }, j_{\min }+1, \ldots, j_{\max }\right\}$ is repeated for 
specified $\mathbf{j}$. They can be calculated explicity by repeated application of the elementary rule for the addition of two angular momenta, as described in detail in [L]. The CG number is shape independent; that is, $N_{j}(\mathbf{j})$ counts the number of orthonormal basis vectors in the basis set $\left\{\left|T\left(\mathbf{j}_{\pi} \mathbf{k}\right)_{j m}\right\rangle \mid\right.$ for specified $\left.j m\right\}$ of the space $\mathcal{H}(\mathbf{j}, j, m)$ for each binary tree $T \in \mathbb{T}_{n}$ and each $j_{\pi}, \pi \in S_{n}$.

It may seem highly redundant to introduce such a variety of orthonormal basis sets of vectors that span the same space $\mathcal{H}_{\mathbf{j}}$, but it is within these vector space structures that resides the entire theory of $3(n-1)-j$ coefficients. This aspect of the theory is realized through shape transformations applied to the binary trees whose standard labels appear in the binary coupled state vectors in the various basis sets $\mathcal{H}_{T}\left(\mathbf{j}_{\pi}, j, m\right), T \in \mathbb{T}_{n}, \pi \in S_{n}$. The implementation of such shape transformations into numerical-valued transformations between such state vectors uses the notion of a recoupling matrix.

The matrix with elements that give the real orthogonal transformation matrix between distinct sets $\left\{\left|T\left(\mathbf{j}_{\pi} \mathbf{k}\right)_{j m}\right\rangle \mid \mathbf{k} \in \mathbb{K}_{T}^{(j)}\left(\mathbf{j}_{\pi}\right)\right\}$ and $\left\{\left|T\left(\mathbf{j}_{\pi^{\prime}} \mathbf{k}^{\prime}\right)_{j m}\right\rangle \mid \mathbf{k} \in \mathbb{K}_{T}^{(j)}\left(\mathbf{j}_{\pi^{\prime}}\right)\right\}$ of simultaneous basis eigenvectors, each of which is an orthonormal basis of the vector space $\mathcal{H}(\mathbf{j}, j, m)$, is called a recoupling matrix. Thus, we have that

$$
\left|T^{\prime}\left(\mathbf{j}_{\pi^{\prime}} \mathbf{k}^{\prime}\right)_{j m}\right\rangle=\sum_{\mathbf{k} \in \mathbb{K}_{T}^{(j)}\left(j_{\pi}\right)}\left(R^{S ; S^{\prime}}\right)_{\mathbf{k}, j ; \mathbf{k}^{\prime}, j}\left|T\left(\mathbf{j}_{\pi} \mathbf{k}\right)_{j m}\right\rangle,
$$

where the recoupling matrix is the real orthogonal matrix, denoted $R^{S ; S^{\prime}}$, with elements given by the inner product of state vectors:

$$
\left(R^{S ; S^{\prime}}\right)_{\mathbf{k}, j ; \mathbf{k}^{\prime}, j}=\left\langle T\left(\mathbf{j}_{\pi} \mathbf{k}\right)_{j m} \mid T^{\prime}\left(\mathbf{j}_{\pi^{\prime}} \mathbf{k}^{\prime}\right)_{j m}\right\rangle .
$$

Here we have written the labeled shapes in the abbreviated forms:

$$
S=S h_{T}\left(\mathbf{j}_{\pi}\right), \quad S^{\prime}=S h_{T^{\prime}}\left(\mathbf{j}_{\pi^{\prime}}\right) .
$$

As this notation indicates, these transformations are independent of $m$; that is, they are invariants under $S U(2)$ frame transformations. The matrix elements are fully determined in each coupling scheme in terms of known generalized WCG coefficients, since each coupled state vector in the inner product is expressed as a linear combination of the orthonormal basis vectors in the set $\mathbf{B}_{\mathbf{j}}$ of the tensor product space $\mathcal{H}_{\mathbf{j}}$ with coefficients that are generalized WCG coefficients. Each recoupling matrix is a fully known real orthogonal matrix of order $N_{j}(\mathbf{j})$ in terms of its elements, the generalized WCG coefficients. Since the inner product is real, it is always the case that the recoupling matrix satisfies the relation

$$
R^{S ; S^{\prime}}=\left(R^{S^{\prime} ; S}\right)^{t r}
$$


where $t r$ denotes the transpose of the matrix. But the most significant property of a recoupling matrix that originates from the completeness of the mutually commuting Hermitian operators that define each binary coupled state is the multiplication property:

$$
R^{S_{1} ; S_{3}}=R^{S_{1} ; S_{2}} R^{S_{2} ; S_{3}}
$$

which holds for arbitrary binary trees $T_{1}, T_{2}, T_{3} \in \mathbb{T}_{n}$ and for all possible labeled shapes $S_{1}=S h_{T_{1}}\left(\mathbf{j}_{\pi^{(1)}}\right), S_{2}=S h_{T_{2}}\left(\mathbf{j}_{\pi^{(2)}}\right), S_{3}=S h_{T_{3}}\left(\mathbf{j}_{\pi^{(3)}}\right)$, where each permutation $\pi^{(i)} \in S_{n}$. It is this elementary multiplication rule that accounts fully for what are known as the Racah sum-rule between Racah coefficients and the Biedenharn-Elliott identity between Racah coefficients. Indeed, when iterated, this multiplication rule generates infinite classes of relations between $3(n-1)-j$ coefficients, $n \geq 3$.

We can now bring together the notion of general shape transformations as realized in terms of words in the elementary association actions $A$ and commutation actions $C$ to arrive at the fundamental relation underlying the properties of the set of binary coupled angular momenta state vectors:

Let

$$
w(A, C)=L_{1} L_{2} \cdots L_{r}
$$

where each $L_{h}$ is either an elementary association operation $A$ or a elementary commutation operation $C$, give a word $w(A, C)$ that effects the shape transformation given by

$$
S_{1} \stackrel{L_{1}}{\longrightarrow} S_{2} \stackrel{L_{2}}{\longrightarrow} S_{3} \stackrel{L_{3}}{\longrightarrow} \cdots \stackrel{L_{r}}{\longrightarrow} S_{r+1} \text {. }
$$

The abbreviated shapes are defined by $S_{h}=S h_{T_{h}}\left(\mathbf{j}_{\pi^{(h)}}\right), h=1,2, \ldots, r$, with corresponding elementary shape transformations given by

$$
S_{h} \stackrel{L_{h}}{\longrightarrow} S_{h+1}, h=1,2, \ldots, r .
$$

Thus, the transformation from the initial shape $S_{1}$ to the final shape $S_{r+1}$ is effected by a succession of elementary shape transformations via $S_{2}, S_{3}, \ldots, S_{r}$. The matrix elements of the product of recoupling matrices given by

$$
R^{S_{1} ; S_{r+1}}=R^{S_{1} ; S_{2}} R^{S_{1} ; S_{2}} R^{S_{2} ; S_{3}} \cdots R^{S_{r} ; S_{r+1}}
$$

is equal to the inner product of binary coupled state vectors given by

$$
\left(R^{S_{1} ; S_{r+1}}\right)_{\mathbf{k}^{(1)}, j ; \mathbf{k}^{(r+1)}, j}=\left\langle T_{1}\left(\mathbf{j}_{\pi^{(1)}} \mathbf{k}^{(1)}\right)_{j m} \mid T_{r+1}\left(\mathbf{j}_{\pi^{(\mathbf{r}+\mathbf{1})}} \mathbf{k}^{(r+1)}\right)_{j m}\right\rangle .
$$

Then, the main result is: There exists such a shape transformation by elementary shape operations between every pair of specified initial and final shapes, $S_{1}$ and $S_{r+1}$. 
We introduce yet another very useful notation and nomenclature for the matrix elements of a general recoupling matrix $R^{S ; S^{\prime}}$, which are given by the inner product of state vectors above. This is the concept of a triangle coefficient, which encodes the detailed coupling instructions of its labeled forks discussed above. A triangle coefficient has a lefttriangle pattern and a right-triangle pattern. The left-triangle pattern is a $3 \times(n-1)$ matrix array whose $1 \times 3$ columns, $n-1$ in number, are the quantum numbers that encode the elementary addition of two angular momenta of the $n-1$ labeled forks that constitute the fully labeled binary tree $T\left(\mathbf{j}_{\pi} \mathbf{k}\right)_{j}$. For example, the column corresponding to the labeled fork 1 given earlier is $\operatorname{col}\left(j_{1} j_{2} k\right)$ with a similar rule for labeled forks of the other three types. The left-triangle pattern of a triangle coefficient is this collection of $n-1$ columns, read off the fully labeled binary tree, and assembled into a $3 \times(n-1)$ matrix pattern by a standard rule. The righttriangle pattern is the $3 \times(n-1)$ triangular array constructed in the same manner from the fully labeled binary tree $T^{\prime}\left(\mathbf{j}_{\pi^{\prime}} \mathbf{k}^{\prime}\right)_{j}$. These two triangle patterns denoted, respectively, by $\Delta_{T}\left(\mathbf{j}_{\pi} \mathbf{k}\right)_{j}$ and $\Delta_{T^{\prime}}\left(\mathbf{j}_{\pi^{\prime}} \mathbf{k}^{\prime}\right)_{j}$, are now used to define the triangle coefficient of order $2(n-1)$ :

$\left\{\Delta_{T}\left(\mathbf{j}_{\pi} \mathbf{k}\right)_{j} \mid \Delta_{T^{\prime}}\left(\mathbf{j}_{\pi^{\prime}} \mathbf{k}^{\prime}\right)_{j}\right\}=\left(R^{S ; S^{\prime}}\right)_{\mathbf{k}, j ; \mathbf{k}^{\prime}, j}=\left\langle T\left(\mathbf{j}_{\pi} \mathbf{k}\right)_{j m} \mid T^{\prime}\left(\mathbf{j}_{\pi^{\prime}} \mathbf{k}^{\prime}\right)_{j m}\right\rangle$.

The main result for the present discussion is: The triangle coefficients (matrix elements of recoupling matrices) for the matrix elements of the elementary commutation operation $C$ and association operation $A$ in the product of recoupling matrices is a basic phase factor of the form $(-1)^{a+b-c}$ for a $C$-transformation, and a definite numerical object of the form $(-1)^{a+b-c} \sqrt{(2 k+1)\left(2 k^{\prime}+1\right)} W$ for an $A$-transformation, where $W$ is a Racah coefficient. It follows that the matrix elements $\left(R^{S_{1} ; S_{r+1}}\right)_{\mathbf{k}^{(1)}, j ; \mathbf{k}^{(r+1)}, j}$ of the recoupling matrix is always a summation over a number of Racah coefficients equal to the number of associations $A$ that occur in the word $w(A, C)$, where the details of the multiple summations depend strongly on the shapes of the underlying pair of binary trees related by the $A$-transformations.

Each word $w(A, C)=L_{1} L_{2} \cdots L_{r}$ that effects a shape transformation between binary coupling schemes corresponding to a shape $S_{1}$ and $S_{r+1}$ has associated with it a path, which is defined to be

$$
\text { path }=S_{1} \stackrel{L_{1}}{\longrightarrow} S_{2} \stackrel{L_{2}}{\longrightarrow} \cdots \stackrel{L_{r}}{\longrightarrow} S_{r+1} .
$$

But there are many distinct words $w_{1}(A, C), w_{2}(A, C), \ldots$ that effect the same transformation

$$
S_{1} \stackrel{w_{i}(A, C)}{\longrightarrow} S_{r+1}, i=1,2, \ldots
$$


via different intermediate shapes. Hence, there are correspondingly many paths of the same or different lengths between the same given pair of shapes, where the length of a path is defined to be the number of associations $A$ in the path. There is, of course, always a path of minimum length. It is this many-fold structure of paths that gives rise to different expressions of one and the same $3(n-1)-j$ coefficient, as well as to a myriad of relations between such coefficients.

An arbitrary triangle coefficient of order $2(n-1)$ is always expressible as a product of recoupling matrices related by elementary shape transformations that give either simple phase transformations or a triangle coefficient of order four, since irreducible triangle coefficients of order four (those not equal to a phase factor or zero) are always of the form $(-1)^{a+b-c} \sqrt{(2 k+1)\left(2 k^{\prime}+1\right)} W$. Thus, triangle coefficients of order four may be taken as the fundamental objects out of which are built all triangle coefficients. Triangle coefficients provide a universal notation for capturing the structure of all recoupling matrices. They possess some general simplifying structural properties that are inherited from the pair of standard labeled binary trees whose labeled fork structure they encode. For the description of this structure, we introduce the notion of a common fork: Two standard labeled binary trees are said to have a common fork if each binary tree contains a labeled fork having endpoints with the same pair of labels, disregarding order.Then, the left and right patterns of the triangle coefficient corresponding to a pair of standard labeled binary tree with a common fork has a column in its left pattern and one in its right pattern for which the entries in the first two rows are either the same or the reversal of one another: If the order of the labels in the two columns is the same, then the triangle coefficient is equal to the reduced triangle coefficient obtained by removal of the column from each pattern and multiplying the reduced pattern by a Kronecker delta factor in the intermediate quantum labels in row three of the common fork column. A similar reduction occurs should the quantum labels of the common fork be reversed, except now a basic phase factor multiplies the reduced triangle coefficient. Of course, if the resulting reduced triangle coefficient contains still a pair of columns corresponding to a common fork, then a further reduction takes place. This continues until a triangle coefficient of order $2(n-1)$ containing $s$ columns corresponding to $s$ common forks is reduced to a product of basic phase factors times an irreducible triangle coefficient of order $2(n-s)$. An irreducible triangle coefficient is one for which the corresponding pair of labeled binary trees has no common fork - the triangle coefficient has no common columns.

But the structure of irreducible triangle coefficients does not stop here. Each irreducible triangle coefficient defines a cubic graph. In particular, irreducible triangle coefficients of order $2(n-1)$ enumerate all possible "types" of cubic graphs of order $2(n-1)$ that can occur in the binary theory of the coupling of angular momenta. The cubic graph 
$C_{2(n-1)}^{*}$ of an irreducible triangle coefficient of order $2(n-1)$ is obtained by the very simple rule: Label $2(n-1)$ points by the $2(n-1)$ triplets (triangle) of quantum labels constituting the columns of the triangle coefficient, and draw a line between each pair of points that is labeled by a triangle containing a common symbol. This defines a graph with $2(n-1)$ points, $3(n-1)$ lines, with three lines incident on each point, which is the definition of a cubic graph $C_{2(n-1)}^{*}$ of "angular momentum type" of order $2(n-1)$. While cubic graphs do not enter directly into such calculations, they are the objects that are used to classify a given collection of $3(n-1)-j$ coefficients into types.

In summary: We have crafted above a conceptual and graphical framework that gives a uniform procedure for computing all $3(n-1)-j$ coefficients, based in the final step on computing the matrix elements of a recoupling matrix expanded into a product of recoupling matrices corresponding to a path of elementary shape transformations. The reduction process, applied to the matrix elements in this product, then automatically reduces in consequence of common forks to give the desired expression for the matrix elements of an arbitrary recoupling matrix (triangle coefficient), which is equal to the inner product of state vectors fully labeled by the simultaneous eigenvectors of the respective pair of complete sets of $2 n$ commuting Hermitian operators. Moreover, properties of recoupling matrices can be used to generate arbitrarily many relations among irreducible triangle matrices of mixed orders, and expressions for one and the same $3(n-1)-j$ in terms of different coupling schemes. The unification of the binary coupling theory of angular momenta is achieved. There remain, however, unresolved problems such as: A procedure for obtaining paths of minimum length, a counting formula for the number of cubic graphs of order $2(n-1)$ of "angular momentum type," and the physical meaning of the existence of many paths for expressing the same $3(n-1)-j$ coefficient. Chapters 1, 5, and 8 provide more comprehensive details of results presented in this overview.

We have taken the unusual step of presenting this overview here in the Preface so as to have in one place a reasonable statement of the coherence brought to the subject of angular momentum coupling theory by the methods outlined above, unblurred by the intricate steps needed in its implementation.

The binary coupling theory of angular momenta has relevance to quantum measurement theory. Measurements of the properties of composite systems is only at the present time, in sophisticated experimental set-ups, revealing the behavior of systems prepared in an initial definite state that remains unmeasured (undisturbed) until some later time, when a second measurement is made on the system. It is the prediction of what such a second measurement will yield that is at issue. This problem can be phrased very precisely in terms of doubly stochastic matrices 
for binary coupled angular momentum states corresponding to complete sets of commuting Hermitian observables; it is so formulated in Chapters 1 and 5, using the property that there is a unique doubly stochastic matrix of order $N_{j}(\mathbf{j})$ associated with each binary coupled scheme 1 and each binary coupled scheme 2 . The probability of a prepared coupled scheme 1 state being in a measured scheme 2 coupled state is just the (row, column) entry in the doubly stochastic matrix corresponding to these respective coupled states. This is the answer given in the context of conventional nonrelativistic quantum theory. We do not speculate on the meaning of this answer to the holistic aspects of complex (or simple) quantal systems. Rather, throughout this volume, we focus on the detailed development of topics and concepts that relate to the binary coupling theory of angular momenta, as developed in [L], [6], and in this volume, leaving their full interpretation for the future.

In many ways, this portion of the monograph can be considered as a mopping-up operation for an accounting of the binary theory of the coupling of arbitrarily many angular momentum systems within the paradigm of conventional nonrelativistic quantum mechanics, in the sense of Kuhn [35, p.24]. Yet, there is the apt comparison with Complexity Theory as advanced by the Santa Fe Institute - a few simple, algorithmic-like rules that generate an almost unlimited scope of patterns of high informational content. Moreover, binary trees viewed as branching diagrams, are omnipotent as classification schemes for objects of all sorts - their shapes and labelings have many applications going beyond angular momentum systems. The closely related graphs — Cayley's [19] trivalent trees, which originate from a single labeled binary tree - and their joining for pairs of such binary trees define cubic graphs (see [L]). These cubic graphs determine the classification of all binary coupling schemes in angular momentum theory. But they surely extend beyond the context of angular momentum systems in sorting out the diverse patterns of regularity in nature, as discussed briefly in Appendix C.

\section{TOPICAL CONTENTS}

We summarize next the principal topics that constitute the present monograph, and the relevant chapters.

Chapter 1, Chapter 5, Chapter 8. Total angular momentum states (reviewed from $[\mathrm{L}]$ ). The total angular momentum of a physical system is a collective property. The addition of two angular momenta, a problem already solved in the seminal papers in quantum mechanics, is the simplest example, especially for intrinsic internal quantum spaces such as spin space. Here we review the earlier work, emphasizing that the tensor product space in which the angular momentum operators act has the property of all such tensor product spaces: It contains vectors that cannot be obtained as simple products of vectors of the individual parts of the system - tensor product spaces by their very nature are holistic; 
that is, are superpositions of the tensor product of the constituent system state vectors. For the most part, our discussions in Chapters 1 and 5 are a summary of material from the first volume needed for this monograph, but now focused more strongly on the properties of the unitary matrices $Z^{j}\left(U^{(\mathbf{j})}, V^{(\mathbf{j})}\right)$, called recoupling matrices, where $U^{(\mathbf{j})}$ and $V^{(\mathbf{j})}$ are unitary matrices that given the transformation coefficients from the uncoupled basis to the coupled basis that determines the composite system state vectors. These recoupling matrices satisfy the very important multiplication rule:

$$
Z^{j}\left(U^{(\mathbf{j})}, V^{(\mathbf{j})}\right) Z^{j}\left(V^{(\mathbf{j})}, W^{(\mathbf{j})}\right)=Z^{j}\left(U^{(\mathbf{j})}, W^{(\mathbf{j})}\right) .
$$

In this relation, each of the unitary matrices $U^{(\mathbf{j})}, V^{(\mathbf{j})}, W^{(\mathbf{j})}$ gives the transformation coefficients of a complete coupled set of state vectors that are simultaneous eigenvectors of the mutually commuting set of Hermitian operators given by the squares of the constituent angular momenta $\mathbf{J}^{2}(i), i=1,2, \ldots, n$, the squared total angular momentum $\mathbf{J}^{2}$ and its 3 -component $J_{3}$, and an additional set of operators (distinct for each of the three cases), or sets of parameter spaces, that complete the set of state vectors. Each set of coupled state vectors then spans the same tensor product space $\mathcal{H}_{\mathbf{j}}$, and the elements of the recoupling matrices give the transformation coefficients from one coupled basis set to the other, either for the full tensor product space $\mathcal{H}_{\mathbf{j}}$, or well-defined subspaces. It is also the case that each of the three recoupling matrices is a doubly stochastic matrix, each of which has a probabilistic interpretation in exactly the same sense as that for von Neumann's density matrices. Thus, the product rule has implications for measurements carried out on systems described by the state vectors corresponding to complete sets of mutually commuting observables.

The above vector space structures are more comprehensive than the notation indicates. This is because we have suppressed the labels in the $S U(2)$ irreducible multiplet ket vector notation $\left|j_{i} m_{i}\right\rangle \in \mathcal{H}_{j_{i}}$. More generally, these ket vectors are given by $\left|\boldsymbol{\alpha}_{i}, j_{i}, m_{i}\right\rangle$ and constitute a complete set of eigenvectors for the $i$-th system $S_{i}$ of the full system $S$; it is the quantum labels in the sequence $\boldsymbol{\alpha}_{i}$ that originate from the eigenvalues of a complete set of mutually commuting Hermitian operators (or other complete labeling schemes) that includes $\mathbf{J}^{2}(i)$ and $J_{3}(i)$ that give a complete set of eigenvectors of system $S_{i}$, which itself could be a composite system with repeated values of the angular momentum quantum numbers $j_{i}$, as controlled by the labels $\boldsymbol{\alpha}_{i}$. The basic multiplication property of the recoupling matrix still holds under an appropriate adaptation of the notations. The key concept is always completeness, first in the set of mutually commuting observables, and then of the simultaneous eigenvectors. Thus, many complex quantal systems come under the purview of the angular momentum structure of composite systems, as we have outlined above. 
Chapters 2-7. Permutation matrices and related topics. There is, perhaps, no symmetry group more important for all of quantum physics than the group of permutations of $n$ objects - the permutation or symmetric group $S_{n}$. Permutation matrices of order $n$ are the simplest matrix realization of the group $S_{n}$ by matrices containing a single 1 in each row and column: They consist of the $n$ ! rearrangements of the $n$ columns of the identity matrix of order $n$. But in this work the symmetric group makes its direct appearance in a different context than the Pauli principle; namely, through Birkhoff's [8] theorem that proves the existence of a subset of the set of all $n$ ! permutation matrices of order $n$ such that every doubly stochastic matrix of order $n$ can be expanded with positive real coefficients in terms of the subset of permutations matrices. We not only present this aspect of doubly stochastic matrices, but develop a more general theory in Chapter 3 of matrices having the same fixed linesum for all rows and columns. Such matrices include doubly stochastic matrices, magic squares, and alternating sign matrices, which are all of interest in physical theory, as discussed in [L]. Here, additional results of interest are obtained, with Chapters 5, 6, 7 being dedicated to each topic, respectively. We comment further on Chapter 5.

Chapter 5. Doubly stochastic matrices. These matrices are introduced in Chapter 1, and the development of further properties continued here. Recoupling matrices introduced in Chapter 1 are doubly stochastic matrices. Such matrices have a probabilistic interpretation in terms of preparation of states corresponding to complete sets of commuting Hermitian operators. The matrix elements in a given (row, column) of a doubly stochastic matrix is the probability of a prepared eigenstate (labeled by the row) of a complete set of mutually commuting Hermitian operators being the measured eigenstate (labeled by the column) of a second (possibly the same) complete set of mutually commuting Hermitian operators. Here, these eigenstates are taken to be the coupled states corresponding to standard labeled binary trees of order $n$. This aspect of doubly stochastic matrices is illustrated numerous times. To my knowledge, doubly stochastic matrices were introduced into quantum theory by Alfred Landé [39], the great atomic spectroscopist from whom I had the privilege of hearing first-hand, while a graduate student at The Ohio State University, his thesis that they are fundamental objects underlying the meaning of quantum mechanics.

Chapter 8. Heisenberg's magnetic ring. This physical problem, addressed in depth by Bethe [5] in a famous paper preceding the basic work by Wigner [82] on angular momentum theory and Clebsch-Gordan coefficients is a very nice application of binary coupling theory. The Hamiltonian comes under the full purview of composite systems: Its state vectors can be classified as eigenvectors of the square of the total angular momentum with the usual standard action of the total angular momentum. The exact solutions are given for composite systems 
containing $n=2,3,4$ constituents, each part with an arbitrary angular momentum. Remarkably, this seems not to have been noticed.

For $n \geq 5$, the magnetic ring problem can be reduced to the calculation of the eigenstates originating from the diagonalization by a real orthogonal matrix of a real symmetric matrix of order equal to the Clebsch-Gordan number $N_{j}(\mathbf{j})$, which gives the number of occurrences (multiplicity) of a given total angular momentum state in terms of the angular momenta of the individual constituents. This Hamiltonian matrix of order $N_{j}(\mathbf{j})$ is a real symmetric matrix uniquely determined by certain recoupling matrices originating from binary couplings of the constituent angular momenta. It is an exquisitely complex implementation of the uniform reduction procedure for the calculation of all $3(n-1)-j$ coefficients that arise; this procedure is itself based on paths, shape transformations, recoupling matrices, and their reduction properties. This approach to the Heisenberg ring problem gives a complete and, perhaps, very different viewpoint not present in the Bethe approach, especially, since no complex numbers whatsoever are involved in obtaining the energy spectrum, nor need be in obtaining a complete set of orthonormal eigenvectors. The problem is fully solved in the sense that the rules for computing all $3(n-1)-j$ coefficients that enters into the calculation of the Hamiltonian matrix can be formulated explicitly. Unfortunately, it is almost certain that the real orthogonal matrix required to diagonalize the symmetric Hamilton matrix, with its complicated $3(n-1)-j$ type structure, cannot be determined algebraically. It may also be the case that numerical computations of the elements of the symmetric Hamiltonian matrix are beyond reach, except for simple special cases. Of course, complex phase factors do enter into the classification of the eigenvectors by the cyclic invariance group $C_{n}$ of the Hamiltonian, but this may not be necessary for many applications.

Finally, there are three Appendices A, B, and C that deal with issues raised in the main text. Appendix $\mathrm{C}$, however, presents natural generalizations of binary tree classifications to other problems, especially, to composite systems where the basic constituents have $U(n)$ symmetry.

\section{MATTERS OF STYLE, READERSHIP, AND RECOGNITION}

On matters of readership and style, we repeat portions of the first volume, since they still prevail.

The very detailed Table of Contents serves as a summary of topics covered. The readership is intended to be advanced graduate students and researchers interested in learning of the relation between symmetry and combinatorics and of challenging unsolved problems. The many examples serve partially as exercises, but this monograph is not a textbook. It is hoped that the topics presented promote further and more rigorous developments. While we are unable to attain the eloquence of Mac Lane [51], his book has served as a model. 
We mention again, as in [L], some unconventional matters of style. We present significant results in italics, but do not grade and stylize them as lemmas and theorems. Such italicized statements serve as summaries of results, and often do not merit the title as theorems. Diagrams and figures are integrated into the text, and not set aside on nearby pages, so as to have a smooth flow of ideas. Our informality of presentation, including proofs, does not attain the status of rigor demanded in more formal approaches, but our purpose is better served, and our objectives met, by focusing on algorithmic, constructive methods, as illustrated by many examples.

As with the earlier volume, this continuing work is heavily indebted to the two volumes on the quantum theory of angular momentum published with the late Lawrence C. Biedenharn [6]. The present volume is much more limited in scope, addressing special topics left unattended in the earlier work, as well as new problems.

Our motivation and inspiration for working out many details of binary coupling theory originates from the great learning experiences, beginning in the early 1990's, as acquired in combinatorial research papers with William Y. C. Chen, which were strongly encouraged by the late Gian-Carlo Rota, and supplemented by his many informative conversations and inspirational lectures at Los Alamos. We do not reference directly many of the seminal papers by Gian-Carlo Rota, his colleagues, and students in this volume, but these publications were foundational in shaping the earlier volume, and are ever-present here. We acknowledge a few of these again: Désarménien, et al. [23]; Kung and Rota [36]; Kung [37]; Roman and Rota [68]; Rota [70]-[71]; Rota, et al. [72]; as well as the Handbook by Graham, et al. [28]. This knowledge acquisition has continued under the many invitations by William Y. C. Chen, Director, The Center for Combinatorics, Nankai University, PR China, to give lectures on these subjects to students and to participate in small conferences. These opportunities expanded at about the same time in yet another direction through similar activities, organized by Tadeusz and Barbara Lulek, Rzeszòw University of Technology, Poland, on Symmetry and Structural Properties of Condensed Matter, under the purview over the years by Adam Mickiewics University, Poznań, University of Rzeszów, and Rzeszów University of Technology. The interaction with Chinese and Polish students and colleagues has been particularly rewarding. Finally, the constant encouragement by my wife Marge and son Tom provided the friendly environment for bringing both the first and second volumes to completion.

Editors Zhang Ji and Lai Fun Kwong deserve special mention and thanks for their encouragement and support of this project.

James D. Louck 


\section{Contents}

Preface and Prelude vii

Notation $\quad$ xxxiii

1 Composite Quantum Systems 1

1.1 Introduction . . . . . . . . . . . . . . . 1

1.2 Angular Momentum State Vectors of a Composite System 4

1.2.1 Group Actions in a Composite System . . . . . . . 10

1.3 Standard Form of the Kronecker Direct Sum . . . . . . . 11

1.3.1 Reduction of Kronecker Products . . . . . . . . . . 12

1.4 Recoupling Matrices . . . . . . . . . . . . . . 14

1.5 Preliminary Results on Doubly Stochastic Matrices and Permutation Matrices ... . . . . . . . . . . 19

1.6 Relationship between Doubly Stochastic Matrices and Density Matrices in Angular Momentum Theory . . . . . 22

2 Algebra of Permutation Matrices 25

2.1 Introduction . . . . . . . . . . . . . . 25

2.2 Basis Sets of Permutation Matrices . . . . . . . . . 31

2.2 .1 Summary . . . . . . . . . . . . . . . . . 41

3 Coordinates of $A$ in Basis $\mathbb{P}_{\Sigma_{n}(e, p)} \quad 43$

3.1 Notations . . . . . . . . . . . . . . . 43 
3.2 The $A$-Expansion Rule in the Basis $\mathbb{P}_{\Sigma_{n}(e, p)} \ldots \ldots \ldots$

3.3 Dual Matrices in the Basis Set $\Sigma_{n}(e, p) \ldots \ldots . \ldots 47$

3.3.1 Dual Matrices for $\Sigma_{3}(e, p) \ldots \ldots \ldots$. . . . 48

3.3.2 Dual Matrices for $\Sigma_{4}(e, p) \ldots \ldots$. . . . 50

3.4 The General Dual Matrices in the Basis $\Sigma_{n}(e, p) \ldots \ldots 3$

3.4.1 Relation between the $A$-Expansion and Dual

Matrices . . . . . . . . . . . . . . 55

4 Further Applications of Permutation Matrices 59

4.1 Introduction . . . . . . . . . . . . . . 59

4.2 An Algebra of Young Operators . . . . . . . . . . . . 60

4.3 Matrix Schur Functions . . . . . . . . . . . 63

4.4 Real Orthogonal Irreducible Representations of $S_{n} \ldots$. . 67

4.4.1 Matrix Schur Function Real Orthogonal Irreducible

Representations . . . . . . . . . . . 67

4.4.2 Jucys-Murphy Real Orthogonal Representations 69

4.5 Left and Right Regular Representations of Finite Groups 72

5 Doubly Stochastic Matrices in Angular Momentum Theory

5.1 Introduction . . . . . . . . . . . . . . . 81

5.2 Abstractions and Interpretations _. . . . . . . . . 89

5.3 Permutation Matrices as Doubly Stochastic . . . . . . . 91

5.4 The Doubly Stochastic Matrix for a Single System with Angular Momentum J . . . . . . . . . . . . . . . 92

5.4 Spin-1/2 System . . . . . . . . . . . . . . 92

5.4.2 Angular Momentum-j System . . . . . . . . . . 94

5.5 Doubly Stochastic Matrices for Composite Angular

Momentum Systems . . . . . . . . . . . . 97

5.5.1 Pair of Spin-1/2 Systems _. . . . . . . . . . 97

5.5.2 Pair of Spin-1/2 Systems as a Composite System . 99

5.6 Binary Coupling of Angular Momenta . . . . . . . . . . . 104 
5.6.1 Complete Sets of Commuting Hermitian

Observables . . . . . . . . . . . . . . . . 104

5.6.2 Domain of Definition $\mathbb{R}_{T}(\mathbf{j}) \ldots \ldots$. . . . . 106

5.6.3 Binary Bracketings, Shapes, and Binary Trees . . . 109

5.7 State Vectors: Uncoupled and Coupled . . . . . . . . . . 115

5.8 General Binary Tree Couplings and Doubly Stochastic

Matrices . . . . . . . . . . . . . . . . 140

5.8 .1 Overview .................... 140

5.8 .2 Uncoupled States . . . . . . . . . . . . . . . . . 142

5.8.3 Generalized WCG Coefficients . . . . . . . . . 143

5.8.4 Binary Tree Coupled State Vectors . . . . . . . . . 145

5.8.5 Racah Sum-Rule and Biedenharn-Elliott Identity as Transition Probability Amplitude Relations . . 153

5.8.6 Symmetries of the $6-j$ and $9-j$ Coefficients . . 165

5.8.7 General Binary Tree Shape Transformations . . . . 167

5.8 .8 Summary . . . . . . . . . . . . . . . . 172

5.8.9 Expansion of Doubly Stochastic Matrices into

Permutation Matrices . . . . . . . . . . . . 174

$\begin{array}{lll}6 & \text { Magic Squares } & 177\end{array}$

6.1 Review . . . . . . . . . . . . . . . . . . . 177

6.2 Magic Squares and Addition of Angular Momenta . . . 180

6.3 Rational Generating Function of $H_{n}(r) \ldots \ldots$. . . 186

7 Alternating Sign Matrices $\quad 195$

7.1 Introduction . . . . . . . . . . . . . . . 195

7.2 Standard Gelfand-Tsetlin Patterns . . . . . . . . . . 197

7.2.1 A-Matrix Arrays . . . . . . . . . . . . . . . . 199

7.2.2 Strict Gelfand-Tsetlin Patterns . . . . . . . . . . . 202

7.3 Strict Gelfand-Tsetlin Patterns for $\lambda=(n n-1 \cdots 21) \quad$. 202

7.3.1 Symmetries . . . . . . . . . . . . 204 
7.4 Sign-Reversal-Shift Invariant Polynomials . . . . . . . . 206

7.5 The Requirement of Zeros . . . . . . . . . . . . . . 211

7.6 The Incidence Matrix Formulation . . . . . . . . . . . 219

\section{The Heisenberg Magnetic Ring}

8.1 Introduction . . . . . . . . . . . . . . . . . . . . 223

8.2 Matrix Elements of $H$ in the Uncoupled and Coupled Bases

8.3 Exact Solution of the Heisenberg Ring Magnet for $n=2,3,4 \ldots \ldots \ldots \ldots \ldots \ldots$

8.4 The Heisenberg Ring Hamiltonian: Even $n$. . . . . . 235

8.4.1 Summary of Properties of Recoupling Matrices . . 240

8.4.2 Maximal Angular Momentum Eigenvalues . . . . . 242

8.4.3 Shapes and Paths for Coupling Schemes I and II . 243

8.4.4 Determination of the Shape Transformations . . . 245

8.4.5 The Transformation Method for $n=4 \ldots 249$

8.4.6 The General $3(2 f-1)-j$ Coefficients . . . . . . 253

8.4.7 The General $3(2 f-1)-j$ Coefficients Continued . 255

8.5 The Heisenberg Ring Hamiltonian: Odd $n$. . . . . . . 261

8.5.1 Matrix Representations of $H$. . . . . . . . 266

8.5.2 Matrix Elements of $R^{\mathbf{j}_{2} ; \mathbf{j}_{1}}$ : The $6 f-j$ Coefficients 269

8.5.3 Matrix Elements of $R^{\mathbf{j}_{3} ; \mathbf{j}_{1}}$ : The $3(f+1)-j$

Coefficients . . . . . . . . . . 276

8.5.4 Properties of Normal Matrices . . . . . . . . . 287

8.6 Recount, Synthesis, and Critique . . . . . . . . . 289

8.7 Action of the Cyclic Group . . . . . . . . . . . . . 292

8.7.1 Representations of the Cyclic Group . . . . . . . 295

8.7.2 The Action of the Cyclic Group on Coupled State

Vectors . . . . . . . . . . . . . . 299

8.8 Concluding Remarks . . . . . . . . . . . . . . . . . . 304 
A Counting Formulas for Compositions and Partitions 305 A.1 Compositions . . . . . . . . . . . . . 305 A.2 Partitions . . . . . . . . . . . . 307

B No Single Coupling Scheme for $n \geq 5$ B.1 No Single Coupling Scheme Diagonalizing $H$ for $n \geq 5$. . 313

C Generalization of Binary Coupling Schemes $\quad 317$ C.1 Generalized Systems . . . . . . . . . . . . . 317 C.2 The Composite $U(n)$ System Problem . . . . . . . . . 321

Bibliography

Index

335

Errata and Related Notes 
This page intentionally left blank 


\title{
Notation
}

\author{
General symbols \\ , \\ $\mathbb{R} \quad$ real numbers \\ $\mathbb{C} \quad$ complex numbers \\ $\mathbb{P} \quad$ positive numbers \\ $\mathbb{Z} \quad$ integers \\ $\mathbb{N} \quad$ nonnegative integers \\ $\mathbb{R}^{n} \quad$ Cartesian $n$-space \\ $\mathbb{C}^{n} \quad$ complex $n$-space \\ $\mathbb{E}^{n} \quad$ Euclidean $n$-space \\ $O(n, R) \quad$ group of real orthogonal matrices of order $n$ \\ $S O(n, \mathbf{R}) \quad$ group of real, proper orthogonal matrices of order $n$ \\ $U(n) \quad$ group of unitary matrices of order $n$ \\ $S U(n) \quad$ group of unimodular unitary matrices of order $n$ \\ $G L(n, \mathbf{C}) \quad$ group of complex nonsingular matrices of order $n$ \\ $\mathbf{M}_{n \times n}^{p}\left(\alpha, \alpha^{\prime}\right)$ set of $n \times n$ matrix arrays with nonnegative \\ elements with row-sum $\alpha$ and column-sum $\alpha^{\prime}$ \\ $\times \quad$ ordinary multiplication in split product \\ $\oplus \quad$ direct sum of matrices \\ $\otimes$ \\ tensor product of vector spaces, Kronecker (direct) \\ product of matrices \\ $\delta_{i, j} \quad$ the Kronecker delta for integers $i, j$ \\ $\delta_{A, B} \quad$ the Kroneker delta for sets $A$ and $B$ \\ $K(\lambda, \alpha) \quad$ the Kostka numbers \\ $c_{\mu \nu}^{\lambda}$ \\ $\operatorname{Par}_{n}$ \\ $\lambda, \mu, \nu$ \\ $|\mathbb{A}|$ \\ the Littlewood-Richardson numbers \\ set of partitions having $n$ parts, including 0 as a part \\ partitions in the set $\operatorname{Par}_{n}$ \\ $[\mathrm{n}]$ \\ cardinality of a set $\mathbb{A}$ \\ set of integers $\{1,2, \ldots, n\}$
}

Specialized symbols are introduced as needed in the text; 
the list below contains a few of the more general ones:

$\begin{array}{ll}\mathbf{J}(i) & \begin{array}{l}\text { angular momentem of constituent } \\ i \in[n] \text { of a composite system }\end{array} \\ \mathbf{K}(i) & \begin{array}{l}\text { intermediate angular momentem } \\ i \in[n-2] \text { of a composite system } \\ \text { total angular momentem of all constituents }\end{array} \\ \mathbf{J} & \begin{array}{l}\text { of a composite system } \\ \text { sequence }\left(j_{1}, j_{2}, \ldots, j_{n}\right) \text { of quantum numbers } \\ \text { of the constituents of a composite system }\end{array} \\ \mathbf{j} & \text { set of binary bracketings of order } n \\ \mathbb{B}_{n} & \text { set of binary trees of order } n \\ \mathbb{T}_{n} & \text { shape of a binary tree } T \in \mathbb{T}_{n}\end{array}$

$S h_{T}(\mathbf{j}) \quad$ shape of a standard labeled binary tree $T \in \mathbb{T}_{n}$ $w(A, C) \quad$ word in the letters $A$ and $C$

$\left|T(\mathbf{j} \mathbf{k})_{j m}\right\rangle \quad$ simultaneous eigenvector of a complete set of $2 n$ angular momentum operators $\mathbf{J}^{2}(i), i=1,2, \ldots, n ; \mathbf{J}^{2}, J_{3}$; $\mathbf{K}^{2}(i), i=1,2, \ldots, n-2$ - also called binary coupled state vectors

$$
\begin{array}{r}
\left\langle T(\mathbf{j} \mathbf{k})_{j m} \mid T^{\prime}\left(\mathbf{j}^{\prime} \mathbf{k}^{\prime}\right)_{j m}\right\rangle \\
\text { inner product of two binary coupled state vectors }
\end{array}
$$

$\mathcal{H}_{\mathbf{j}_{i}}$

$\mathcal{H}_{\mathbf{j}}=$

$N_{j}(\mathbf{j})$

Hilbert vector space of dimension $2 j_{i}+1$ that is irreducible under the action of $S U(2)$

$\mathcal{H}_{\mathbf{j}_{1}} \otimes \mathcal{H}_{\mathbf{j}_{2}} \otimes \cdots \otimes \mathcal{H}_{\mathbf{j}_{n}}$ : tensor productof the spaces $\mathcal{H}_{\mathbf{j}_{i}}$ of dimension $\left(2 j_{1}+1\right) \cdots\left(2 j_{n}+1\right)=N(\mathbf{j})$

$\mathcal{H}(\mathbf{j}, j, m)$

Clebsch-Gordan number

$U^{\dagger} V$

subspace of $\mathcal{H}_{\mathbf{j}}$ of order $N_{j}(\mathbf{j})$

$R^{S ; S^{\prime}}$

Landé form of a doubly stochastic matrix

recoupling matrix for a pair of standard labeled

binary trees related by arbitrary shapes $S$ and $S^{\prime}$

$R^{S_{h} ; S_{h+1}}$

recoupling matrix for a pair of standard labeled

binary trees of shapes $S_{h}$ and $S_{h+1}$ related by

an elementary shape transformation

$\left\{\Delta_{T}(\mathbf{j} \mathbf{k})_{j} \mid \Delta_{T^{\prime}}\left(\mathbf{j}^{\prime} \mathbf{k}^{\prime}\right)_{j}\right\}$

triangle coefficient that is a $3 \times(n-1)$

matrix array that encodes the structure of the

labeled forks of a pair of standard labeled binary trees 
$\mathbb{G}_{\lambda}$

$\left(\begin{array}{c}\lambda \\ m\end{array}\right)$

$E(x)$

$e_{i j}(x)$

$\mathcal{P}_{n}$

$\mathbb{P}_{\Sigma_{n}}$

$\mathbb{P}_{\Sigma_{n}(e)}$

$\mathbb{P}_{\Sigma_{n}(e, p)}$

$\mathbb{A}_{n}$

$\mathbb{M}_{n}(r)$

$\mathbb{A} S_{n}$

$l_{A}$
Gelfand-Tsetlin (GT) pattern of shape $\lambda$

member of $\mathbb{G}_{\lambda}$

linear matrix form

element of $E(x)$

vector space of linear forms

basis set $\Sigma_{n}$ of permutation matrices

basis set $\Sigma_{n}(e)$ of permutation matrices

basis set $\Sigma_{n}(e, p)$ of permutation matrices

set of doubly stochastic matrices of order $n$

set of magic squares of order $n$ and line-sum $r$ set of alternating sign matrices of order $n$ line-sum of a matrix $A$ of fixed line-sum 